\title{
Anisotropy Searches with the High-Resolution Fly's Eye
}

J. Belz ${ }^{\mathrm{a}}$, for the HiRes Collaboration

a Physics Department, University of Utah

Salt Lake City UT 84112, U.S.A.

We present here the results of new searches for anisotropy within the HiRes data set, including both monocular and stereo events with energies exceeding $1 \mathrm{EeV}$. We include the results of searches for pointlike excesses, including correlations with BL-Lacertae objects, as well as extended sources and dipole moments in the full-sky survey.

\section{Introduction}

The High-Resolution Fly's Eye (HiRes) has been summarized for this conference by P. Sokolsky [1]. Here, we provide greater detail on the results of various searches for arrival direction anisotropy that have been carried out using the HiRes data.

I present the results of studies using both the monocular and stereo data sets.

\section{Monocular Summary}

Although the monocular data set contains the greatest statistical power of HiRes data sets, it has a relatively large uncertainty in the track angle within the shower-detector plane (the angle $\Psi$ in Figure 1). This can be resolved by the use of a timing fit

$t_{i}=t_{o}+\frac{R_{P}}{c} \tan \frac{\theta_{i}}{2}$

where the $t_{i}$ represent the hit times of the various photomultipliers recording the fluorescence signal. In the case of shorter tracks (particularly the HiRes-I detector, consisting of one ring of mirrors covering from $3^{\circ}$ to $16.5^{\circ}$ in elevation angle) this fit is supplemented by constraining the shower profile to conform to the Gaisser-Hillas function [2].

The resulting fits yield ellipticial arrival direction uncertainties, as illustrated in Figure 2. Nevertheless, the monocular data is still sensitive to pointlike sources (Figure 3). Although they will not be discussed in greater detail here, HiRes

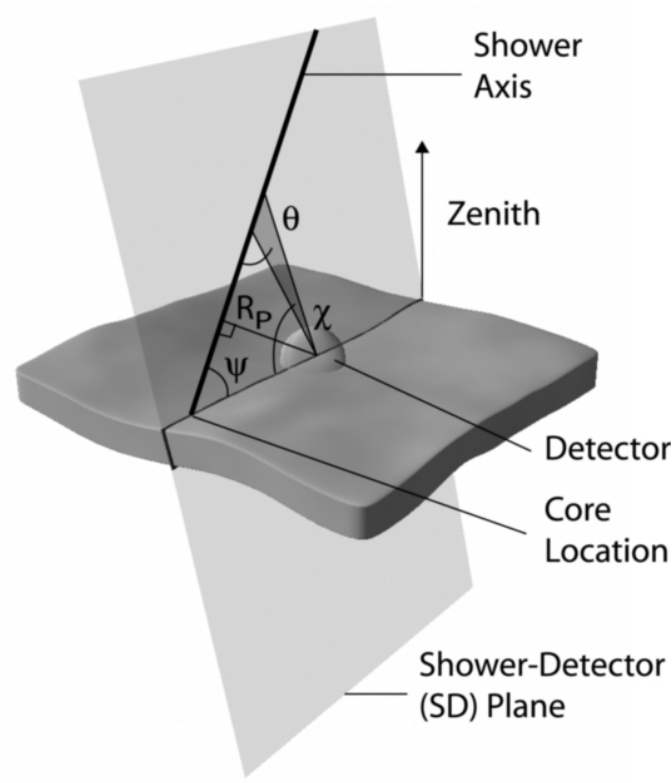

Figure 1. The geometry of monocular reconstruction of extensive air showers.

has published several null results in monocular searches for pointlike arrival direction excesses. These include autocorrelation studies [5], point (neutral particle) source searches [6], and searches for cross-correlations with the AGASA doublets and triplet [7].

HiRes has also published null results in searches for dipole enhancement in the direction of the Galactic Center, Centaurus A, and M87 [8]. 


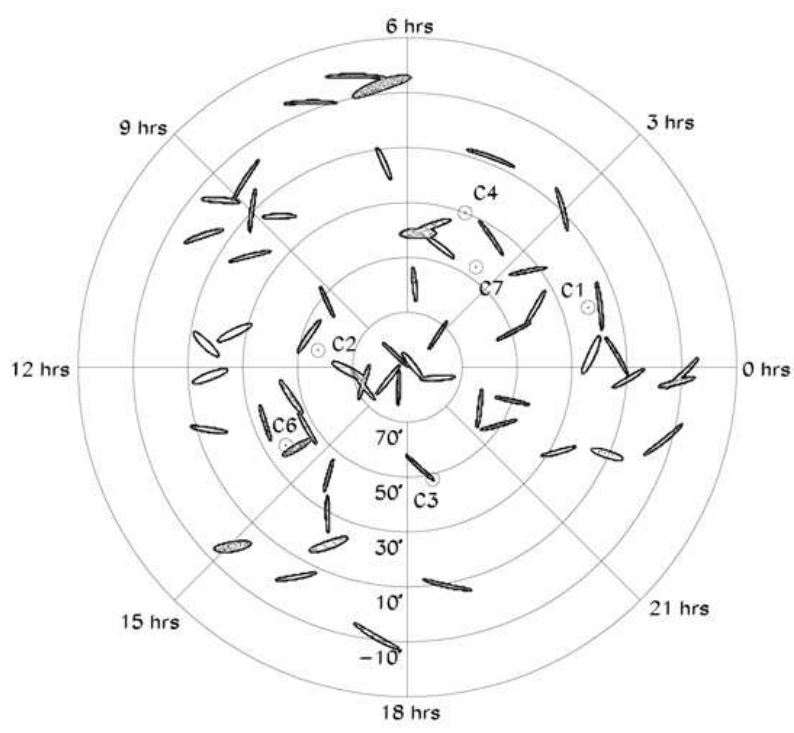

Figure 2. Arrival directions of HiRes-I monocular events above $4 \times 10^{19} \mathrm{eV}$, plotted on a polar projection, equatorial coordinates. Each event is represented by an ellipse representing 1- $\sigma$ reconstruction uncertainty. Also shown are the positions of the six AGASA clusters C1-C5 and C7 [3,4].

\section{Stereo Results}

On a per-event basis, stereo reconstruction provides far better resolution of airshower arrival directions. Each HiRes site determines a shower detector plane, and shower geometry is determined by a global $\chi^{2}$-minimization using the pointing and timing information of all photomultiplier tubes. In Monte Carlo simulations, $68 \%$ of events above $10 \mathrm{EeV}$ are reconstructed within $0.6^{\circ}$ of their true arrival direction (Figure 4). From star surveys and reconstruction of Rayleigh-scattered laser light, we estimate that systematic uncertainties are less than $0.6^{\circ}$, mainly caused by uncertainties in mirror pointing directions.

The skymaps for the HiRes stereo data sets are shown in Figure 5.

\subsection{Stereo Point Source Search}

The stereo data was used to perform a maximum likelihood point source search, as follows.

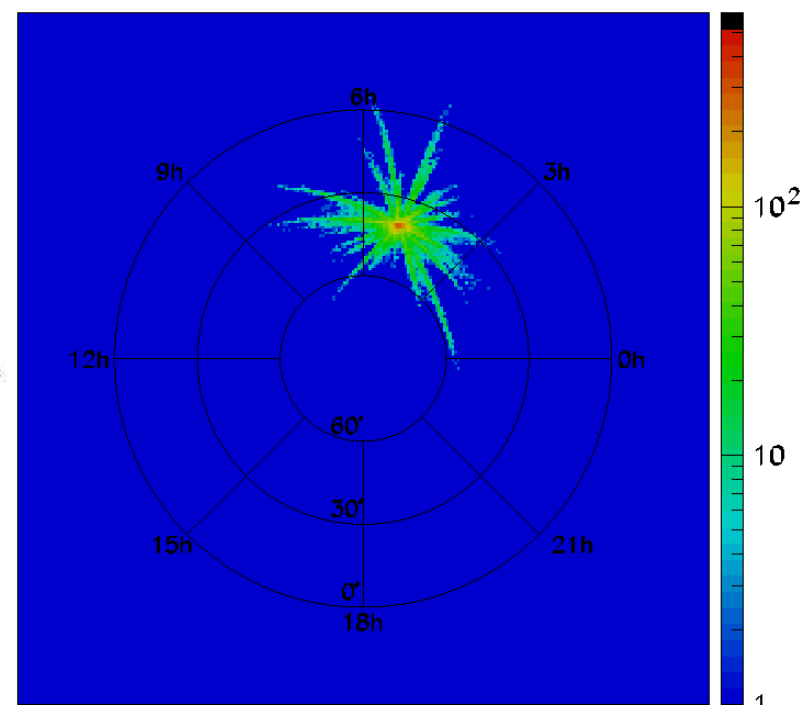

Figure 3. Event detection probability (arbitrary, logarithmic scale) for a simulated pointlike source of cosmic rays.

We define $Q_{i}\left(x_{i}, x_{s}\right)$ as the probability for an event observed at $x_{i}$ to have a true arrival direction at $x_{s}$, and $R_{i}(x)$ as the probability distribution for the event to be observed anywhere in the sky. $R_{i}$ depends on the detector acceptance and exposure.

The probability associated with a given event, under the point source hypothesis, is the weighted sum $P_{i}$ of the source and background probabilities.

$P_{i}\left(x, x_{s}\right)=\frac{n_{s}}{N} Q_{i}\left(x, x_{s}\right)+\frac{N-n_{s}}{N} R_{i}(x)$

The product of the $P_{i}$ for all events gives the likelihood $L$ for a particular choice of the number of source events $n_{s}$. The best estimate for $n_{s}$ is the value which maximizes $L$ :

$L\left(n_{s}, x_{s}\right)=\prod_{i=1}^{N} P_{i}\left(x, x_{s}\right)$

In practice, we maximize $\ln R$, the $\log$ of the ratio of the likelihood of $n_{s}$ relative to the likelihood of the null hypothesis $n_{s}=0$

$\ln R=\ln \frac{L\left(n_{s}, x_{s}\right)}{L\left(0, x_{s}\right)}$ 


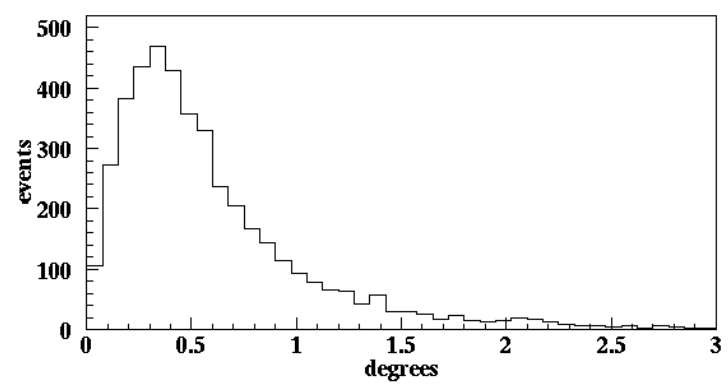

Figure 4. Magnitude of difference between true and reconstructed arrival direction for simulated HiRes stereo events above $10^{19} \mathrm{eV}$.

$\ln R$ is the measure of deviation from the null hypothesis of no source events.

Now, given a set of data, we scan over a fine grid of locations in the sky treating each as a source position and identify the single spot in the skymap with the highest $\ln R$. The significance is determined by scanning over Monte Carlo data sets and counting the fraction with $\ln R_{M C}>\ln R_{D A T A}$.

In Figure 6 we illustrate the results of performing the above analysis on the combined HiRes stereo and AGASA [3] data set above $40 \mathrm{EeV}$. The highest value of $\ln R=8.54$, corresponding to $N_{S}=2.9$, at the location of the AGASA triplet. The fraction of Monte Carlo sets with greater $\ln R$ is $28 \%$. We conclude that no significant point source is found in the combined set of HiRes stereo and AGASA events above $40 \mathrm{EeV}$.

We find that if the HiRes threshold is lowered to $30 \mathrm{EeV}$, an additional event is observed in the vicinity of the triplet (Figure 7 ). The highest value of $\ln R=12.98$, and the fraction of Monte Carlo sets with greater $\ln R$ is $0.5 \%$. However, this result contains some biases: (1) The clustered AGASA events which were used to establish the $40 \mathrm{EeV}$ threshold are still included in the sample, and (2) the HiRes energy threshold has to be changed to include an event that contributes to the cluster. These biases imply that $0.5 \%$ should be interpreted as a lower bound on the chance probability [10].
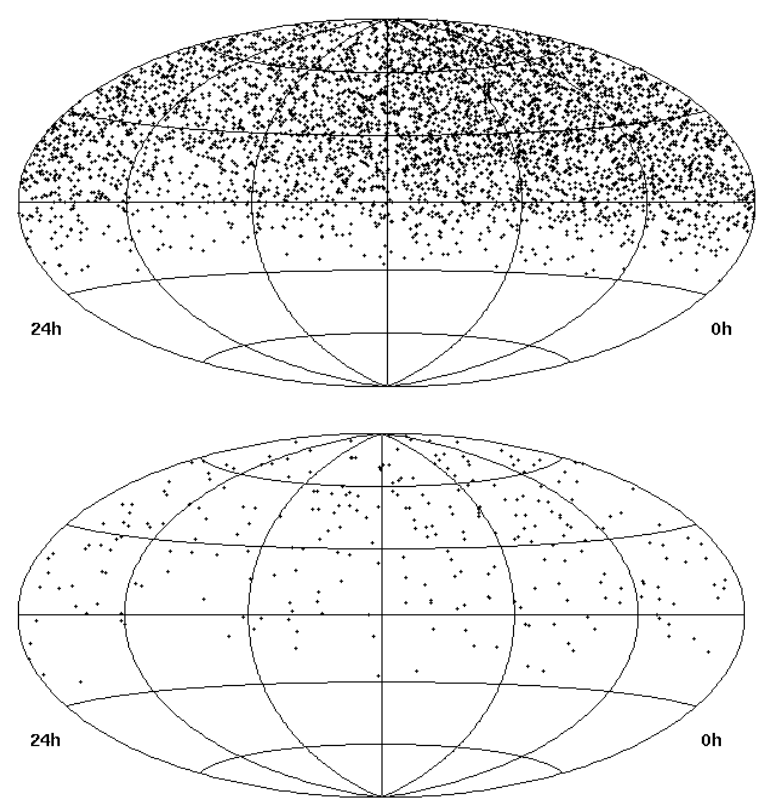

Figure 5. Top: The skymap for the HiRes stereo data set, including airshowers of all energies above $10^{18.2} \mathrm{eV}$ collected between December 1999 and January 2004 (4495 events). Bottom: The skymap for the HiRes stereo data set, including airshowers of energies above $10^{19} \mathrm{eV}$ (271 events). This skymap was used in producing the HiRes stereo autocorrelation null result of Reference [9].

\subsection{Correlations with BL-Lac Objects}

BL Lacertae objects are believed to comprise a special class of blazars, in which the jet axis of the active galaxy is aligned with our line of sight. Blazars are established sources of $\mathrm{TeV} \gamma$ rays, which may be produced in the acceleration of EeV energy cosmic rays. Recently, a series of articles have claimed evidence for correlations between UHECR and BL-Lac objects [11-17].

We first address the claims made using data from other experiments, which HiRes is in a position to confront with a statistically independent sample of cosmic rays. Table 1 lists the results of BL-Lac correlation searches made with data from the AGASA and Yakutsk arrays. While the re- 


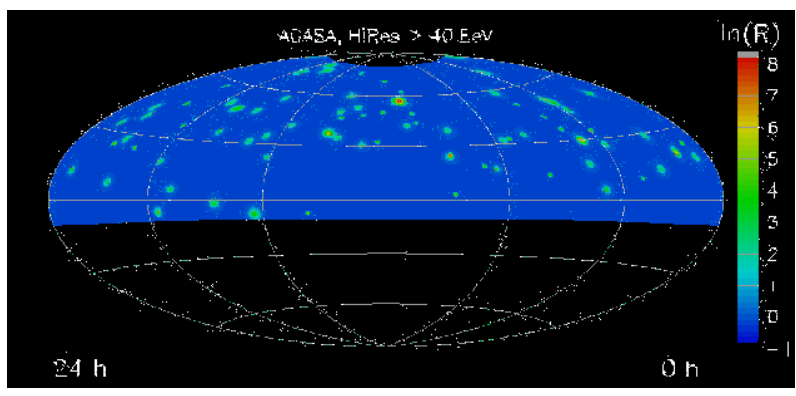

Figure $6 . \ln R$ for the combined HiRes stereo and AGASA data set above $40 \mathrm{EeV}$. The location of the AGASA "triplet" stands out as having the highest log-likelihood.

ported significance of the previous claims show low probability of arising from isotropic background, an identical analysis performed with the HiRes data shows no significant correlation above background expectations.

More recently, Gorbunov et al. [16] have carried out a similar analysis using the HiRes stereo data above $10 \mathrm{EeV}$ (Figure 5). Using all BL-Lacs with magnitude $m<18$ in the 10th Veron Catalog [20] (156 objects) and an optimized bin size of $0.8^{\circ}$, Gorbunov et al. found 10 correlated BLLac cosmic ray pairs, with a chance probability of 0.001 .

To verify this claim, we prefer to perform an unbinned maximum likelihood analysis as described in the previous section, modified for a multiple-source hypothesis [21]. The results of this analysis indicate an excess of $n_{s}=8.0$ events correlating with BL Lacs. Only $2 \times 10^{-4}$ of isotropic Monte Carlo data sets score a stronger signal. We are therefore in agreement with Gorbunov et al. that the observed correlation is "interesting".

However since we are uncertain as to what tuning of cuts was applied in finding the reported correlation, we believe that any such claims must be tested with independent data. Fortunately, new data is available within HiRes: Arrival directions have not been examined for the data taken between January 2004 and the turn-off of HiRes in April 2006. This data set is approximately $70 \%$

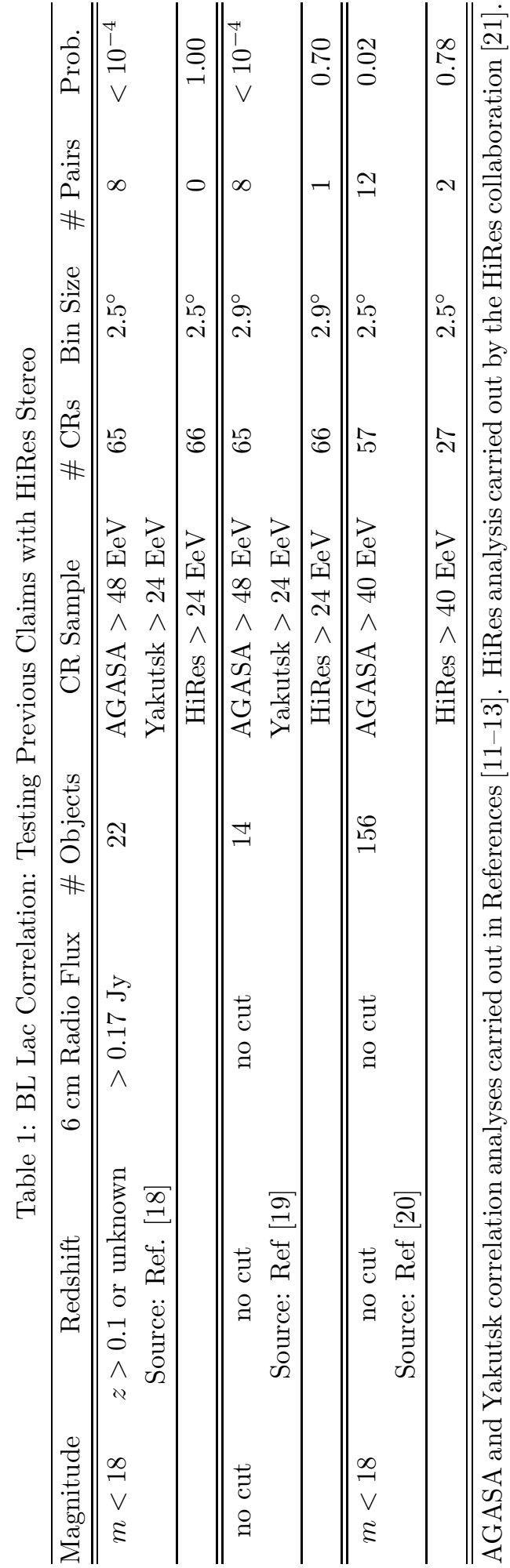




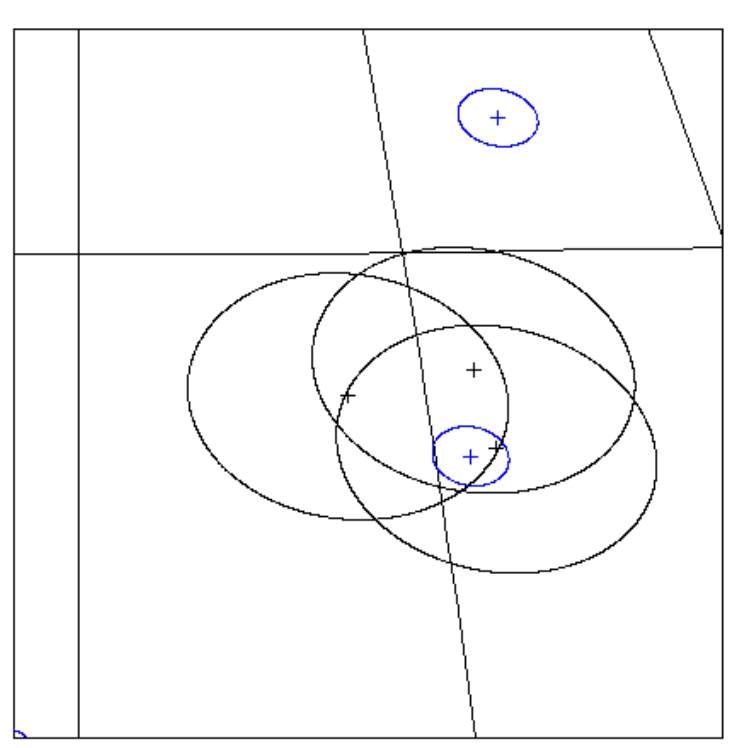

Figure 7. Vicinity of AGASA triplet, Threshold lowered to $30 \mathrm{EeV}$ for HiRes events (blue). An additional HiRes event overlaps the triplet. See text for a discussion of the significance of this observation.

of the current sample size. A future publication will address this important analysis.

\section{Large-Scale Anisotropy}

In 1999, AGASA reported a large excess of cosmic rays in the 1-2 EeV energy band in the region near the galactic center and Cygnus [4]. This result (Figure 8) may also be interpreted as a dipole, owing to the observed deficit in the direction of the galactic anti-center. These claims need to be verified by an independent experiment.

For the HiRes analysis, we have concluded that the usual technique of "time-swapping" is not effective for large scale effects. Instead, to take into account seasonal fluctuations in exposure to the sky (Figure 9) we use the detector livetime in the integration of isotropic background. That is, we assume the rate at which cosmic rays strike the earth is constant at different sidereal times and

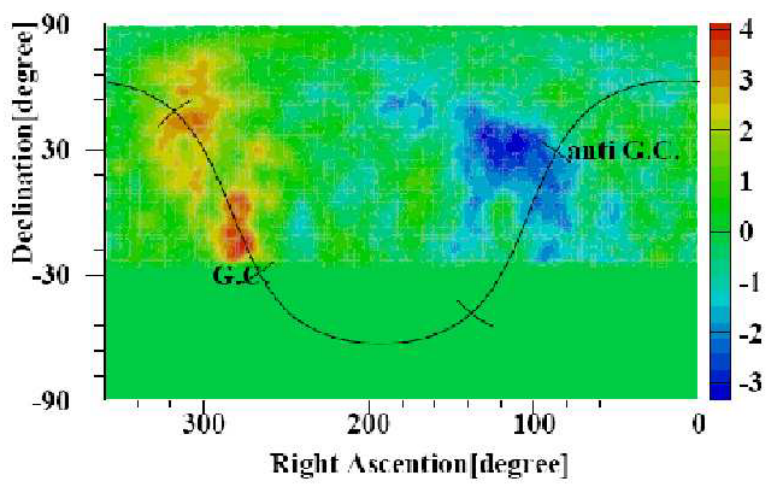

Figure 8. AGASA skymap results [4], events with energies in the 1-2 EeV band.

throughout the year.

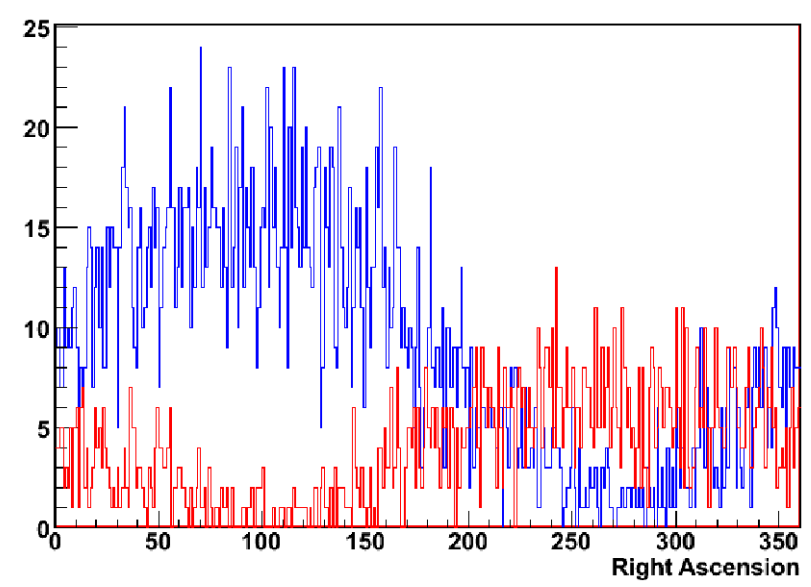

Figure 9. Histogram of collected cosmic rays, during the summer (red) and winter (blue) halves of the year.

To show the effectiveness of livetime integration in uncovering large-scale effects, we inject a signal

$S(R A)=1.5-\sin (R A)$

into a Monte Carlo data set. Then, we assume an exposure of

$E(R A)=1.5+\sin (R A)$ 
in order to make the number of observed events constant as a function of sidereal time. Figure 10 illustrates the results of analyzing the resulting data set both ways, via time swapping and livetime integration. Clearly live-time integration is the superior technique for extracting large-scale effects.
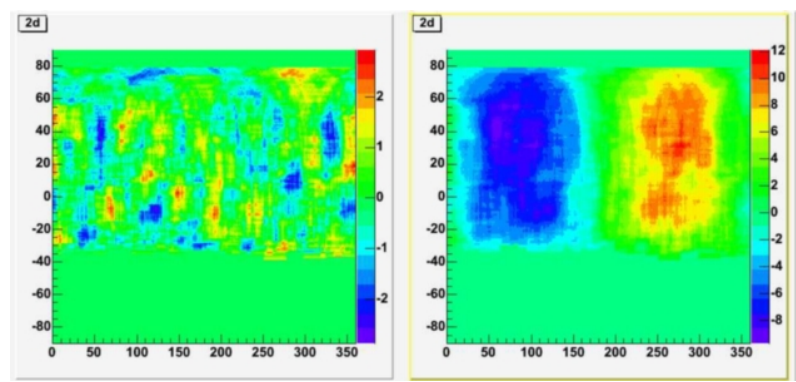

Figure 10. Results of applying the techniques of time swapping (left) and livetime integration (right) to the extraction of a simulated signal, masked by exposure as described in the text.

Next, we apply the livetime integration analysis to the HiRes stereo and monocular data sets. Here, we show only the results of the HiRes-II monocular analysis. This data set, consisting of 10,326 events collected between December 1999 and August 2004 is the HiRes data set most comparable in statistical power to that of the AGASA result. The results of this analysis are shown in Figure 11.

Ideally, we would like to obtain a single number to characterize the significance of the entire map. For now, we quote the RMS of the significance distribution, as compared to the RMS observed in simulated data sets. We find that in $10^{5}$ simulations, $216(0.2 \%)$ have an RMS greater than that observed in the data (1.176). The HiRes monocular data set thus has an effect of low significance (0.002). However, this effect is not observed in the stereo data set, a fact which implies that the observed monocular result is a fluctuation.

We emphasize that the above result should be regarded as preliminary; A test of the effect of

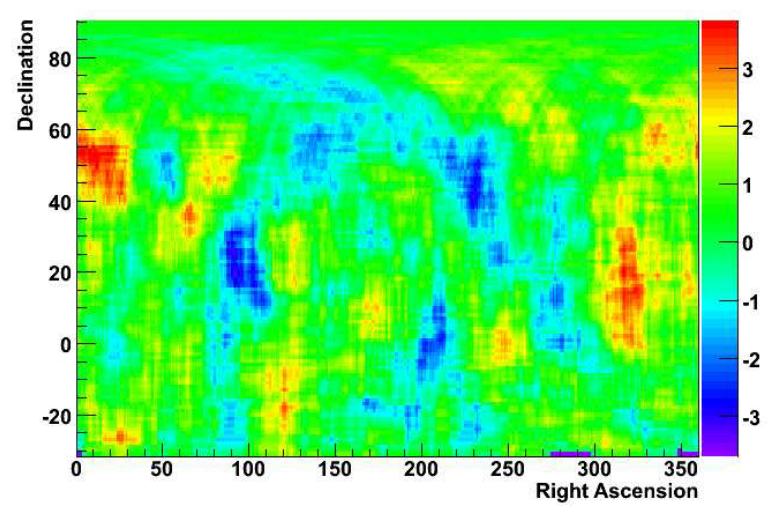

Figure 11. Preliminary results of applying livetime integration analysis to the HiRes-II monocular data set (10,326 events), with 20 degree smoothing. Note that to compare this plot with the AGASA result (Figure 8) it is necessary to "flip" the plot in R.A.

seasonal atmospheric variations on the observed event rate while the detector is "live" is currently in progress.

\section{Conclusions}

Although the HiRes monocular data has elliptical arrival direction uncertainty due to the ambiguity in resolving track angle within the shower-detector plane, it is nevertheless of use in searching for arrival direction anisotropy. Previous HiRes results enumerated above, as well as a new preliminary result in the search for large-scale anisotropy highlight the importance for anisotropy searches of the most statistically powerful of HiRes data sets.

HiRes' finest resolution view of the sky comes from the stereo data set. We have presented the results of searches for point sources, including likelihood comparisons with AGASA and BL Lac objects. As in the case of the monocular data, no clear evidence for signal has been observed. 


\section{REFERENCES}

1. P. Sokolsky, proceedings of this conference.

2. T.K. Gaisser and A.M. Hillas, Proc. 15th ICRC 8, 353 (1977).

3. N. Hayashida et al., Phys. Rev. Lett. 77, 1000 (1996)

M. Takeda et al., Ap.J 522, 225 (1999)

4. N. Hayashida et al. Astropart. Phys. 10, 303 (1999)

N. Hayashida et al. Proc. 26th ICRC 3, 256 (1999).

5. R.U. Abbasi et al., Astropart. Phys. 22, 139 (2004).

6. R.U. Abbasi et al., submitted to Astropart. Phys., astro-ph/0507663.

7. J. Belz, Proc. $29^{\text {th }}$ ICRC (2005).

8. R.U. Abbasi et al., Astropart. Phys. 21, 111 (2004).

9. R.U. Abbasi et al., ApJ 610, L73 (2004).

10. R.U. Abbasi et al., ApJ 623, 164 (2005).

11. Tinyakov and Tkachev, JETP 74445 (2001).

12. Tinyakov and Tkachev, Astropart. Phys. 18 165 (2002).

13. Gorbunov et al., ApJ 577, L93 (2002).

14. Evans, Ferrer and Sarkar, Phys. Rev. D67 103005 (2003).

15. Torres et al., ApJ 595 L13 (2003).

16. Gorbunov et al., JETP Lett 80145 (2004).

17. Stern and Poutanen ApJ 623 L33 (2005).

18. M. P. Véron-Cetty and P. Véron, ESO scientific report (2000).

19. M. P. Véron-Cetty and P. Véron, Astron. Astrophys. 37492 (2001). BL Lacs correlated with EGRET sources were considered.

20. ibid. All BL Lac objects were considered.

21. R.U. Abbasi et al. ApJ 636, 680 (2006). 